\title{
Web GIS to Identify the Problematic Mobile Signal Clusters
}

\author{
Amila Karunathilake \\ Postgraduate Institute of \\ Science, \\ University of Peradeniya, \\ Sri Lanka
}

\author{
M.A.P. Chamikara \\ Department of Statistics and \\ Computer science, \\ Faculty of Science, \\ University of Peradeniya, \\ Sri Lanka
}

\author{
Jagath Gunatilake \\ Postgraduate Institute of \\ Science, \\ University of Peradeniya, \\ Sri Lanka
}

\begin{abstract}
Mobile communications has become one of the fastest growing sectors in the world today. With the technological advancement, mobile communication has subjected to many upgrades such as 2G, 3G, 4G. The question of "Does a customer get the expected capabilities from it?" is not answered yet. Even though, the subscribers of all operators pay almost equal charges per minute, most of the time, they do not get the real benefit from the service. At the moment there is no any location based system to capture the availability of signal receiving levels (specially $3 \mathrm{G}$ and $4 \mathrm{G}$ signal for Dongles), when a customer sit in front of the marketing person, asking to provide a new connection. What most of the customers do is to use the equipment for few days and return them with a complaint of malfunction in case of signal unavailability. In this study geostatistical analysis was carried out by the method of Inverse distance weighting and the interpolated maps were generated using ArcMap 10. Maps were uploaded to the map server, with standard color ramp. Thereby, the network users can get a better idea about the variation of mobile signal receiving level in a particular location. The developed web based GIS (Geographic Information Systems) system provides the capability of accessing the mobile signal levels remotely in an online manner prior to dealing with a particular customer. Analysis of receiving signal level variation helps to find clusters which have low signal levels than expected. Also, further investigation can be carried out to determine the frequently changing network clusters against a relevant time domain.
\end{abstract}

\section{Keywords}

Geostatistical analysis, Mobile drive test, Mobile networks, Signal receiving level, Web GIS

\section{INTRODUCTION AND OBJECTIVE}

Mobile signals are not in the range of visible spectrum which cannot be observed by the naked eye. Therefore people cannot understand how these mobile signals behave under different conditions making the users struggle when a signal interruption occurs. Visualization and prediction of these signals will provide a remarkable assistance for the people living in a technologically advanced era like the present [1].

Mobile signals transmit in the form of Electro Magnetic (EM) radiation. Therefore, the wave properties such as reflection, refraction, diffraction, interference are applicable for mobile signals also [2]. But the mobile subscribers won't get the expected quality of service even when they reside just close by the base transceiver station [3].

This generates a vast amount of problems to the mobile operators with high a customer disappointment. Background noise, poor signal receiving level and call interruption during traveling are a few of them [3]. Maintaining error free networks with higher number of subscribers and an expected data rates with a better quality have become a challenging task for all most all the mobile operators.

In this study, the mobile signal receiving level of $2 \mathrm{G}$, networks has been considered. Mobile signal receiving level basically depends on the distance to the base transceiver station location which transmits the mobile signal.

But EM wave propagation properties and geographical surface variation make this process very difficult to be predicted properly [3] [4]. Geostatistical analysis with mobile signal receiving level data which is taken from an existing mobile network will provide a reliable analysis for mobile signal receiving level distribution. Visualization in the sense of general color ramp makes it easy to understand about the mobile signal strengths in a precise manner [4]. Google maps base map layer provides a better understanding about the location information of the areas with malfunctioning mobile signal distributions [5].

\section{Objective}

Providing a platform to identify and analyze the mobile network signal distributions which is available in an area of interest. The web based architecture provides an easy remote access to the developed system through the internet. Necessity of a thorough knowledge to analyze and identify the mobile signal level distributions has been minimized to a very lower level by the introduction of this automated system. Up to date coverage information which is provided by the system has increased the quality and the efficiency of decision making.

Most of the time subscribers suffer because of the unavailability of network services from place to place in their busy day today life making them disturbed with poor mobile network services.

But, the proposed system will guarantee a better quality of mobile network service making the customers to build a positive attitude towards the mobile network services. The GIS based web system displays, mobile signal receiving levels with the standard color ramp. Poor receiving level clusters and no service locations are highlighted using different colors helping the user to identify the problematic signal clusters. This will help to optimize network errors for better quality of service.

\section{MATERIALS AND METHODOLOGY 2.1 Materials 2.1.1 Open Layers}


Open Layers is completely free, Open Source JavaScript, released under the 2-clause BSD License (also known as the FreeBSD).It is capable of displaying map tiles and markers loaded from a source map service [5].

\subsubsection{MS4W Map server}

MS4W stands for Map Server for Windows. This is free and open source software package. It allows all levels of map server users to quickly install a working environment for map server development on Windows. Packing and distributing map server applications can be done using MS4W environment [6].

\subsubsection{Web client frame work}

An open source web client framework at OSGEO which was used to implement the web interface.

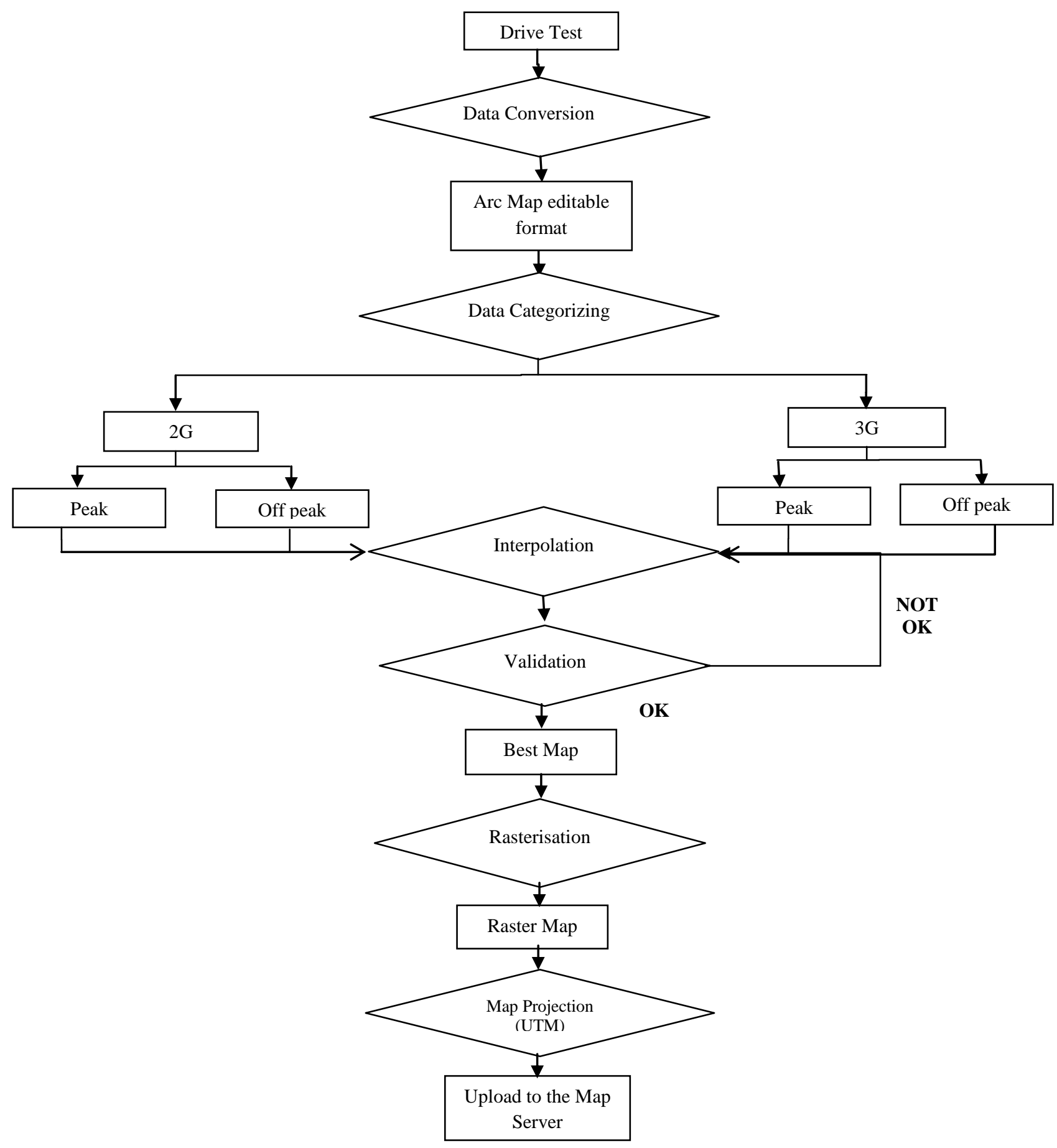

Figure 1: Architecture of the system 
Core of this program was written in JavaScript and HTML. It runs on web servers such as Apache, IIS, and Nginx [7].

\subsubsection{Apache Web server}

Apache is an open-source HTTP server, available basically for the two operating systems UNIX and Windows NT. It provides a secure, an efficient and an extensible server that provides HTTP services in sync with the current HTTP standards [8].

\subsubsection{My $S Q L$}

My SQL is a relational data base management system which is used for adding, removing and modifying information in a particular database [9].

\subsection{Methodology}

\subsubsection{Drive Test}

Mobile signal monitoring process is simply known as drive test [10]. In this process monitoring equipment both hardware and software were implement inside a motor vehicle. Ericsson TEMP 9.0 software was used in recording mobile signal receiving level and an air interface monitoring phone was connected to the laptop to get input in units of $\mathrm{dBm}$ [11]. Bluetooth GPS with a better accuracy is used to record the mobile signal receiving level with its spatial location in WGS84 coordinates.

\subsubsection{Architecture of the System}

Preliminary study was carried out to understand the network traffic of one whole day ( $24 \mathrm{~h})$. After analyzing the Base Transceiver Station (BTS) traffic of 24 hour period, the analysis was divided into two parts. That is peak traffic (PT) time and off peak traffic (OPT) time. By analyzing the 24 hour period, generally two peak hours were observed. So, the drive test time was scheduled according to a predefined time period for PT and OPT. Average value of PT and average value of OPT were analyzed separately. The mobile signal receiving level obviously has a higher value near the base transceiver stations. Therefore, the GPS coordinates of these BTS locations were captured by a hand held GPS device.

It helps to determine the optimum path for the drive test to investigate the problematic clusters.

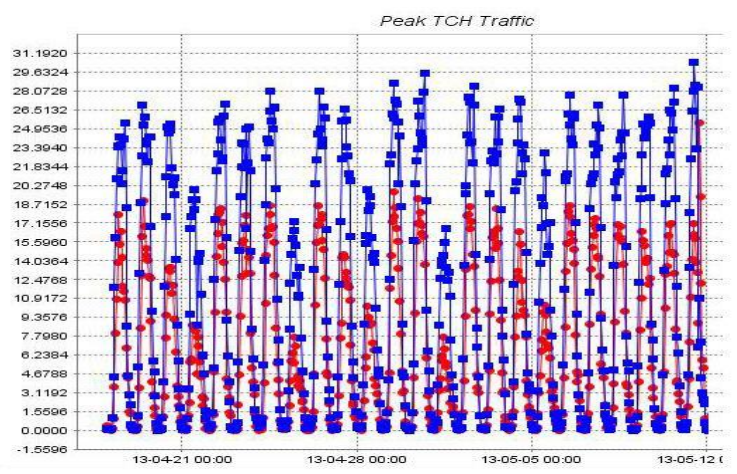

Figure 2: Plot of Peak network traffic Vs. Time

Network monitoring process was done for both $2 \mathrm{G}$ and $3 \mathrm{G}$ mobile networks. Recording relevant mobile signal receiving levels within a relevant time period (peak and off peak hours) is important. Therefore, simultaneous monitoring was carried out using two laptops independently. Drive test track was decided according to the city traffic plan. It helps to record data by traveling a minimum distance while covering a large area at once.
Recorded log files were exported and converted in to tabular format which can be retrieved using Microsoft excel [12]. To prepare the coverage maps files, relevant mobile signal receiving levels and their spatial location longitude and latitude coordinates data was extracted. All the tabular files were inserted to the MySQL database server and a further classification was examined. Data was categorized in to four main types of tables, $2 \mathrm{G}$ peak time period, $2 \mathrm{G}$ off-peak time period, $3 \mathrm{G}$ peak time period, $3 \mathrm{G}$ off peak time period. Then the $2 \mathrm{G}$ and the $3 \mathrm{G}$ mobile signal receiving levels of peak and off peak hours were loaded into separate databases and test points were extracted for the validation purposes. Analysis of the data and validation of the data files of $2 \mathrm{G}$ and $3 \mathrm{G}$ modes were imported in to personal geodatabase of ESRI ArcMap 10.

Overlay analysis was done with city road network which is depicted in Figure 5 for the categorization before the interpolation. Then the clusters were clarified with respect to the covered areas of drive test and tower population.

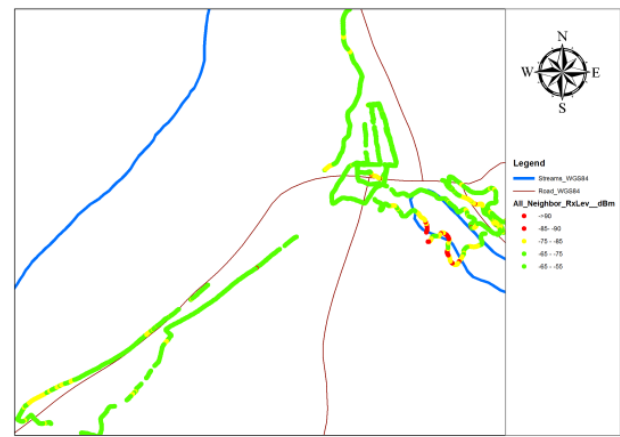

Figure 3: Drive test tracks

\subsubsection{Interpolation}

A geostatistical interpolation was carried out for the determination of the best method to predict mobile signal receiving level. Geostatistical interpolation techniques of kriging and Inverse distance weighting were selected after the first stage of analysis. [4] [13] Selection of these two methods done by considering minimum standard deviation [14]. Different type of kriging patterns of ordinary kriging, simple kriging, universal kriging, indicator kriging were analyzed.

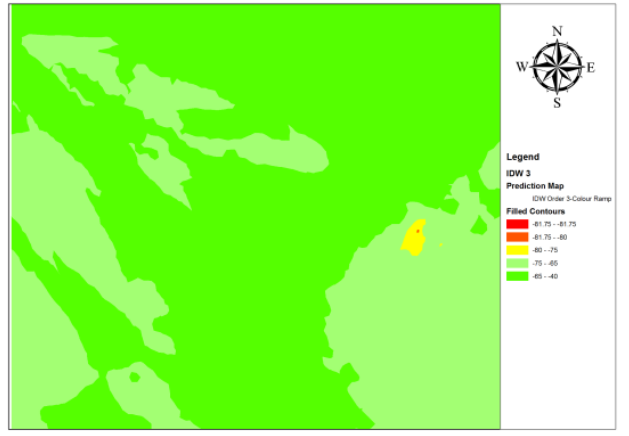

Figure 4: Inverse distance weighting Interpolation

Inverse distance weight with the powers of $1,2,3$ with different neighboring limits were analyzed. Randomly extracted points were used to determine the accuracy of the validation. Method of interpolation was finalized in the final stage by considering the standard deviation value for each and every method mentioned [14]. Inverse distance weighting 
method of power 3 results in the least value for sigma. Then Inverse distance weighting method was finalized as the geostatistical interpolation technique for the study.

\subsubsection{Inverse distance weighting}

A general form of finding an interpolated value $\mathrm{Z}$ at a location $\mathrm{P}$, given values $\mathrm{Z}(1), \ldots, \mathrm{Z}(\mathrm{n})$ at distinct other locations $\mathrm{P}(1), \ldots, \mathrm{P}(\mathrm{n})$, respectively. The interpolated value is a weighted average of the Z's. It develops a sequence of numbers (the "weights") w(1), .., w(n) and forms

$$
Z=\frac{[w(1) * Z(1)+\ldots+w(n) * Z(n)]}{[w(1)+\ldots+w(n)]}
$$

Where

$$
w(i)=\frac{1}{\text { Distance }(P, P(i))^{p}}
$$

power ' $p$ ', is usually around 2,3 . The weights are inversely proportional to the power ' $\mathrm{p}$ ' of the distances between the point of interpolation and the data locations [15].

Total interpolated map area was converted into the raster format. All the raster maps were saved in a map server. It gives quick access to webserver requests which is displayed on top of Google maps [6]. Interpolated maps show a smooth variation of mobile signal strengths in most of the places. But in some places sudden decrements can be observed. Then the raster 'tif' format was used to display the final map file on top of the base Google maps. Colors were assigned according to general color ramp which is used to display corresponding dBm units [11] [16] [17].

Table 1: Mobile signal receiving level classification

\begin{tabular}{|c|c|c|}
\hline $\begin{array}{c}\text { Signal Level } \\
\text { Range(dBm) }\end{array}$ & Classification & Color \\
\hline-120 to -95 & Extremely Bad & \\
\hline-95 to -85 & Bad & \\
\hline-85 to -75 & Average & \\
\hline-75 to -65 & Good & \\
\hline-65 to -55 & Very Good & \\
\hline
\end{tabular}

\section{RESULTS AND DISCUSSION}

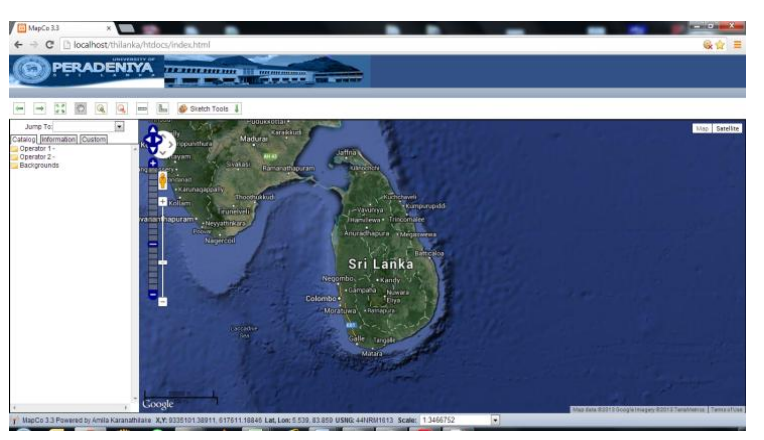

Figure 5 depicts the default web interface of the map sever after login to the system. Left side of the interface contains the main folders which guide to map files. It contains Google maps as the base layer.

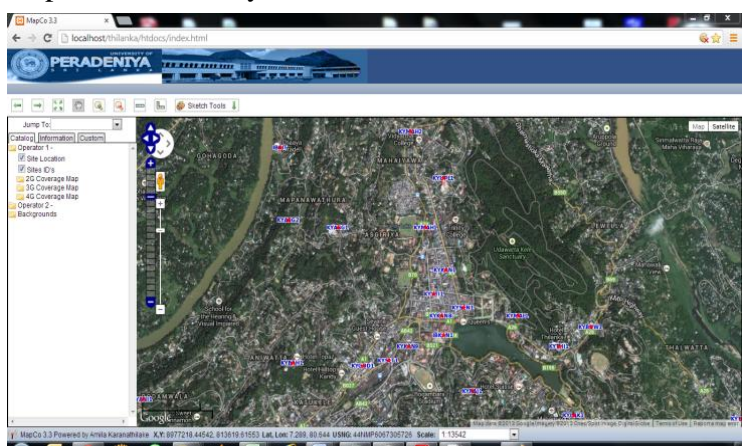

Figure 6: BTS locations with site identification codes

User can see the coordinates in Latitudes, Longitudes or Universal transvers marketer format at the bottom panel of the web interface. Search window with "Jump To" function give quick zoom to the Kandy city area as shown in Figure 6.

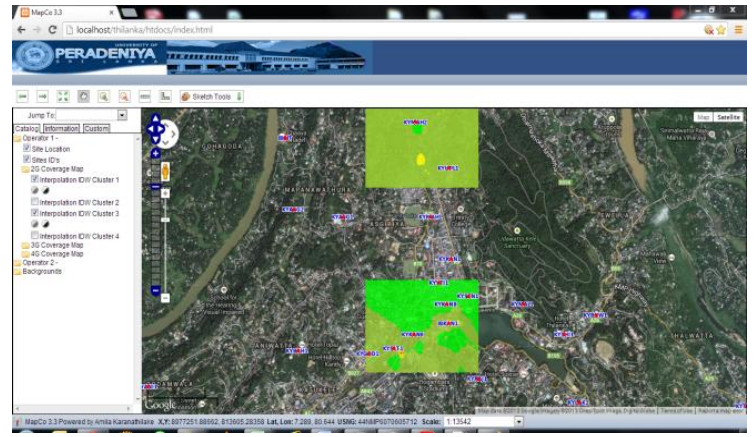

Figure 7: Interpolation of Mobile signal receiving level ( $2 \mathrm{G}$ coverage map)

By a single click of left panel, the system will display the $2 \mathrm{G}$ coverage cluster on top of Google base layer as shown in Figure 7. Transparency of the clusters can also be adjusted using the contrast tool. Users can find their locations and relevant mobile signal receiving levels easily by using the zoom in, zoom out and pan tools. Distance in between two BTS locations or distance to the problematic location can be measured using the measuring tool, provided. It may help to get an idea before adjusting the BTS parameters such as the antenna direction or tilt.

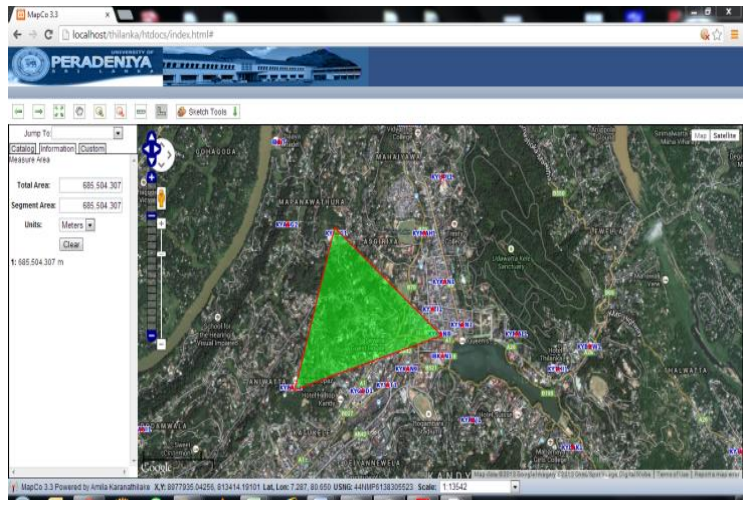

Figure 8: Tool set for overlay analysis

Figure 5: Default Web interface 
Transmission power limitations in between two BTS locations can be decided with the knowledge of the ground area in relevant units as shown in Figure 8. This can also be used to identify the locations which require new BTS for coverage enhancements.

\section{CONCLUSION}

This system will help identifying the problematic mobile signal clusters so that, the network optimization engineers can directly focus their attention on these problematic places depending on the result generating from the proposed system. Subscriber locations and relevant receiving levels give clear understanding about usability of the equipment (specially 3G dongles) or commercial packages such as remote monitoring etc. System can be further developed to monitor all the mobile operators to provide a statistical measurement for their quality of service.

\section{REFERENCES}

[1] Stallings, William.,Data and computer communication .,Chapter 4- Transmission media 4.3 Wireless propagation., $8^{\text {th }}$ edition Copyright 2007 by PrenticeHall,Inc

[2] Tomasi, Wayne.,(2009). Electronic Communications Systems, Fundamentals through advanced., Chapter 14Electromagnetic wave propagation., Fifth edition ISBN 9780130494924., published by Pearson Education, Inc., Copyright 2004

[3] Coverage or Capacity-making the best use of $802.11 \mathrm{n}$ meeting the need for high performance and ubiquitous coverage with the proliferation of mobile devices in the enterprise., white paper., Copyright (C) 2011, Juniper Networks, Inc

[4] Kolyaie, S., Yaghooti, M., Gilda, M., (). Analysis and simulation of wireless signal propagation applying geostatistical interpolation techniques, Archives of Photogrammetry, Cartography and Remote Sensing, Vol. 22, 2011, pp. 261-270, ISSN 2083-2214

[5] Documentation-Open layers. Copyright 2005-2011 Open Layers Contributors,. Accessed on August, 2013. < http://trac.osgeo.org/openlayers/wiki/Documentation>.

[6] Documentation-MS4W., Copyright (C) 1996-2007 Regents of the University of Minnesota, Licenses is free of charge. Accessed on August, 2013. $<\mathrm{http} / / /$ www.maptools.org/ms4w/index.phtml?page $=$ doc s.html>.

[7] Documentation- GeoMOOSE. Copyright () 2009-2012, Dan "Ducky" Little \& GeoMOOSE.org, The GeoMOOSE license is an MIT based license. Accessed on August, 2013.

<http://www.geomoose.org/documentation.html>.

[8] Apache HTTP Server Version 2.2 Documentation., Accessed on January 2004.,Online [August 2013]., <http://httpd.apache.org/docs/2.2/>

[9] What is MySQL?., Accessed on February 2012., <http://dev.mysql.com/doc/refman/4.1/en/what-ismysql.html>

[10] Drive Test, Accessed on October,2013. http://www.3g.co.uk/PR/August2009/First-TD-LTEReceiver-Measurements-Drive-Test-System-3G.html, accessed on July,2013.

[11] What is $0 \mathrm{dBm}$ ?., TelecomHall,online referenc., Posted by leopedrini Friday, $3^{\text {rd }}$ December, 2010, accessed on February 2012., < http://www.telecomhall.com/what-is-0dbm.aspx>

[12] TEMS Logfile., online help aeixo., Accessed on September

2012., <http://www.aexio.com/onlinehelp/xeusug/TEMS FMT_file.htm>

[13] Methods of Generating Surfaces In Environmental GIS Applications., Jun (John) Hu, Bechtel Environmental Inc., 151 Lafayette Drive, Oak Ridge, Tennessee 37922. Accessed on January 2013., <http://proceedings.esri.com/library/userconf/proc 95/to100/p089.html>

[14] Standard deviation., accessed on October 2013. $<$ http://www.mathsrevision.net/gcse-mathsrevision/statistics-handling-data/standard-deviation >

[15] Huber, Bill., "What formula is used in IDW interpolation?". ArcGIS Desktop Discussion Forums, Published on 11 December 2005. Web. Accessed on August 2013 <http://forums.esri.com/Thread.asp?c=93\&f=995\&t=176 542>.

[16] Akram, A., Al-Mashouq.K., R.R. and Hackney, O.P. (2011). QMeter Tools for Quality Measurment inTelecommunication Network, International Junal of Distributed and Parallel Systems (IJDPS). Vol.2, No.4, July 2011

[17] Lehtimaki, P., Raivio, K., Simula, O.,(). Mobile radio access network monitoring using the self-organizing map, ESANN'2002 proceedings - European Symposium on Artificial Neural Networks Bruges (Belgium), 24-26 April 2002, d-side public., ISBN 2930307-02-1, pp. 231-236 